\title{
Behavioral and Pharmacological Validation of the Gerbil Forced-Swim Test: Effects of Neurokinin-I Receptor Antagonists
}

\author{
Tanya L Wallace-Boone*,', Amy E Newton,' Robert N Wright', Nicholas J Lodge' and John F McElroy' \\ 'Neuroscience Drug Discovery, Bristol-Myers Squibb Pharmaceutical Research Institute, Wallingford, CT, USA
}

\begin{abstract}
Several studies have suggested that neurokinin-I (NKI) receptor antagonists may have therapeutic potential as novel antidepressant drugs. To test these compounds preclinically, gerbils have become one of the preferred species in that they demonstrate close NKI receptor homology with humans and bind NKI antagonists with higher affinity than rats and mice. The intent of the present study was to determine whether the forced-swim test (FST), one of the most commonly used animal tests of antidepressant-like activity, could be adapted for use with the gerbil. Critical factors in the establishment of this assay included swim tank diameter, weight, and sex of the animals tested. Pharmacological validation of the FST using standard antidepressant compounds (eg fluoxetine, paroxetine, desipramine) resulted in decreased immobility time during the test, indicative of an antidepressant-like effect. Similar to results reported for the rat and mouse FST, the antipsychotic drug haloperidol increased immobility, whereas the psychostimulant, amphetamine decreased immobility, and anxiolytic drugs (eg buspirone) had no effect. Investigation into the locomotor effects of all compounds tested was consistent with previous reports in other species, with the exception of paroxetine, which produced hyperactivity at therapeutically effective doses in gerbils. In addition to standard antidepressants, NKI antagonists (L-733060, MK-869, and CP-I2272I) all reduced immobility in the gerbil FST without affecting locomotor activity. Overall, these results suggest that the gerbil is an ideal species for use in the FST, and that this paradigm may have predictive validity for identifying novel antidepressant compounds.

Neuropsychopharmacology (2008) 33, 1919-1928; doi: I0.1038/sj.npp. I30 I586; published online 3 October 2007
\end{abstract}

Keywords: Gerbil; depression; forced swim; neurokinin; antidepressant; behavioral model

\section{INTRODUCTION}

Traditionally, one of the difficulties in discovering novel treatments for depression has been in developing and/or optimizing existing animal models for the detection of new mechanisms of action (Nestler et al, 2002). Based on the symptomology of depression (eg anhedonia, helplessness, cognitive deficits), it has been postulated that the complex emotional nature and higher cognitive functioning involved in the disease pathology are specific to the human condition and cannot be modeled overtly in rodents (Wong and Licinio, 2004). However, several acute and chronic models are currently in use that model-specific symptoms of the disorder and have high predictive validity for detecting antidepressant efficacy (Maier and Seligman, 1976; Lucki, 1997; Porsolt et al, 1977a, b; Porsolt et al, 1978a, b; Steru et al, 1985; Varty et al, 2003).

\footnotetext{
* Correspondence: Dr TL Wallace-Boone. Current address: Neuroscience Department, Roche Palo Alto, 343I Hillview Avenue, R2-I0I, Palo Alto, CA 94304, USA, Tel: + 6503547219 , Fax: + 65085231 II, E-mail: tanya.wallace.twl@roche.com

Received 19 January 2007; revised 14 July 2007; accepted 23 August 2007
}

Among the various different animal models currently employed for screening antidepressant compounds, the forced-swim test (FST) is one of the most commonly employed. Initially developed by Porsolt et al (1977a), this behavioral assay is based on the premise that rodents forced to swim in an inescapable environment will eventually minimize all active behaviors and exert only those necessary to keep from drowning. The emergence of the characteristic behavioral despair response, measured as time spent immobile, is selectively suppressed by the administration of all classes of clinically validated antidepressant compounds and is not affected by compounds from other therapeutic classes (eg anxiolytics). Overall, the FST is a useful model for detecting antidepressant-like efficacy with high predictive validity and selectivity. In addition, the FST has an advantage over chronic models (eg chronic mild stress) of being relatively high-throughput and replicable across different laboratories (Detke et al, 1997; Cryan et al, 2002).

Since the original report by Porsolt et al (1977a), the FST has been successfully modified and extended to other species, namely mice (Porsolt et al, 1977b; Lucki et al, 2001; Crowley et al, 2004). In recent years, however, the use of gerbils in modeling psychiatric disorders has garnered 
much attention due in part to the purported involvement of the neurokinin-1 (NK1) receptor in anxiety and depressive disorders (Kramer et al, 1998). Interestingly, the gerbil NK1 receptor shares greater sequence homology with the human NK1 receptor as compared to the rat or mouse counterpart (Beresford et al, 1991; Gitter et al, 1991), thereby making the gerbil an ideal species to test the behavioral effects of NK1 receptor antagonists. Moreover, NK1 antagonists (eg MK869, L-733060) have been shown preclinically to exhibit anxiolytic-like effects in gerbils in several different animal models including the elevated plus maze, fear conditioning, social interaction, and shock-induced foot tapping (Ballard et al, 2001; File et al, 2001; Gentsch et al, 2002; Rupniak et al, 2003; Varty et al, 2002b); and more recently, antidepressant-like effects in the tail suspension test (Varty et al, 2003). Consistent with these reports, genetic deletion of the NK1 receptor in mice resulted in anxiolytic and antidepressant effects in the elevated plus maze, novelty suppressed feeding model and the FST (Santarelli et al, 2001; Rupniak et al, 2001), providing additional support for the hypothesis that the NK1 receptor may be an important target for antidepressant/anxiolytic therapy.

It was the intent of the present studies to determine whether the FST could be modified for use in the gerbil to reliably detect and predict antidepressant-like activity, and to identify the behavioral effects of NK1 antagonists in this paradigm. Several variables, including swim tank diameter, weight, and sex of the animal were important factors in the validation of this assay. In addition, since the effects on immobility in the FST can be influenced by changes in locomotor activity (LMA), all compounds tested in the FST were also examined for their effects on LMA.

\section{METHODS}

\section{Subjects}

Male and female Mongolian gerbils (Charles River Laboratories, Kingston, NY) weighing 45-80 g were used for all studies. The gerbils were housed five per cage in shoebox cages in a colony room maintained at constant temperature $\left(21-22^{\circ} \mathrm{C}\right)$ and humidity $(50 \pm 10 \%)$. The room was illuminated $12 \mathrm{~h}$ per day (lights on at 0600 hours). The gerbils had ad libitum access to food and water throughout the study. All experiments were conducted during the light phase (between the hours of 0700 and 1400 hours). Gerbils were allowed at least 1 week to acclimate to the change in environment before the testing began. All experimental procedures were performed according to protocols approved by the Animal Care and Use Committee of the Bristol-Myers Squibb Company and the published guidelines in the National Institutes of Health Guide for the Care and Use of Laboratory Animals in an Association for Assessment and Accreditation of Laboratory Animal Care (AAALAC)-accredited facility.

\section{Forced-Swim Test}

Naïve gerbils were placed individually in either a 4-1 glass beaker $(15 \mathrm{~cm}$ diameter, $24 \mathrm{~cm}$ height, filled $18-20 \mathrm{~cm}$ ), a $30 \mathrm{~cm}$ diameter plexiglass cylinder $(60 \mathrm{~cm}$ height and filled $21-23 \mathrm{~cm}$ ), a $38 \mathrm{~cm}$ width $\times 48 \mathrm{~cm}$ length $\times 20 \mathrm{~cm}$ height plexiglass rectangle (filled approximately $18 \mathrm{~cm}$ ), or a $50 \mathrm{~cm}$ diameter metal cylinder painted white $(30 \mathrm{~cm}$ height and filled $21-23 \mathrm{~cm}$ ) For all studies, the water temperature was between 24 and $26^{\circ} \mathrm{C}$ and the water was deep enough that the animals could not touch the bottom with their feet or tails. The water was changed between each animal. The total duration of immobility (complete lack of movement or only slight movement needed to stay afloat) was measured during a 6 min test. Unlike mice and rats, gerbils exhibited bouts of immobility that occurred throughout the $6 \mathrm{~min}$ test session, including the first $2 \mathrm{~min}$, thus immobility was measured for the entire 6 min session to ensure the most accurate assessment. Immediately after the test the gerbils were placed in a cage placed on a heating pad to dry.

\section{Locomotor Activity}

LMA was measured using an automated photobeam system (DigiScan Animal activity monitors, Omnitech Electronics Inc., Columbus, Ohio). Animals were placed into the center of a plexiglass LMA chamber and LMA was recorded for $60 \mathrm{~min}$. All activity was recorded by computer using the DigiPro software. D-Amphetamine $(5 \mathrm{mg} / \mathrm{kg}$, p.o.) was used as a positive control with all of the compounds tested.

\section{Drugs}

Compounds were prepared as suspensions in an aqueous vehicle of $0.25 \%$ methylcellulose (methylcellulose, Type AL5c, Dow Chemicals, Midland, MI, USA), except paroxetine which was prepared in distilled water. Suspensions were bead-milled overnight using three layers of $4 \mathrm{~mm}$ glass beads, or sonicated. Compounds were administered orally by gavage (p.o.) $60 \mathrm{~min}$ or $120 \mathrm{~min}$ (NK1 antagonists) before behavioral testing in a volume of $10 \mathrm{ml} / \mathrm{kg}$ body weight. Doses and pretreatment times were selected based on internally generated pharmacokinetic and pharmacodynamic data, as well as from previously published reports in which in vivo efficacy was demonstrated (Porsolt et al, $1977 \mathrm{a}, \mathrm{b}$; Varty et al, 2002b, 2003). Oral administration of compounds was used whenever possible in attempt to validate the model using the anticipated clinical route of administration. Doses of all drugs were calculated and are expressed in terms of the free base weight.

\section{Data Analysis}

Results are expressed as the mean \pm SEM. All data were subjected to one-way anaylsis of variance followed by individual mean comparisons with Fisher's PLSD test using StatView statistical software Version 5.0. The accepted level of significance was set at $p<0.05$.

\section{RESULTS}

\section{Critical Factors in Establishing the Gerbil Forced-Swim Model}

It has been shown previously in mice that the size of the swim tank is a critical factor in detecting antidepressant-like properties of drugs (Sunal et al, 1994). Similarly, increasing the diameter of the swim tank to $50 \mathrm{~cm}$ in the gerbil FST was 
essential for the successful completion of the 6-min test (ie approximately $95 \%$ of gerbils completed the test without needing to be rescued from drowning (Table 1). In comparison, smaller swim tank diameters that were investigated, including $15 \mathrm{~cm}$ and $30 \mathrm{~cm}$ tanks, resulted in only 65 and $19 \%$ successful completion of the 6 min test, respectively. Therefore, all subsequent testing was performed in the $50 \mathrm{~cm}$ diameter swim tank.

Table I Critical Factors for Antidepressant-like Activity

\section{Swim tank dimensions}

\begin{tabular}{lc}
\hline $50 \mathrm{~cm}$ diameter & $\sqrt{ }$ \\
$30 \mathrm{~cm}$ diameter & - \\
$15 \mathrm{~cm}$ diameter & \\
Weight & \\
$\quad \geqslant 55 \mathrm{~g}$ & - \\
$\quad<55 \mathrm{~g}$ & \\
Sex & \\
Male & \\
Female & - \\
\hline
\end{tabular}

$\sqrt{ }=$ critical factor.
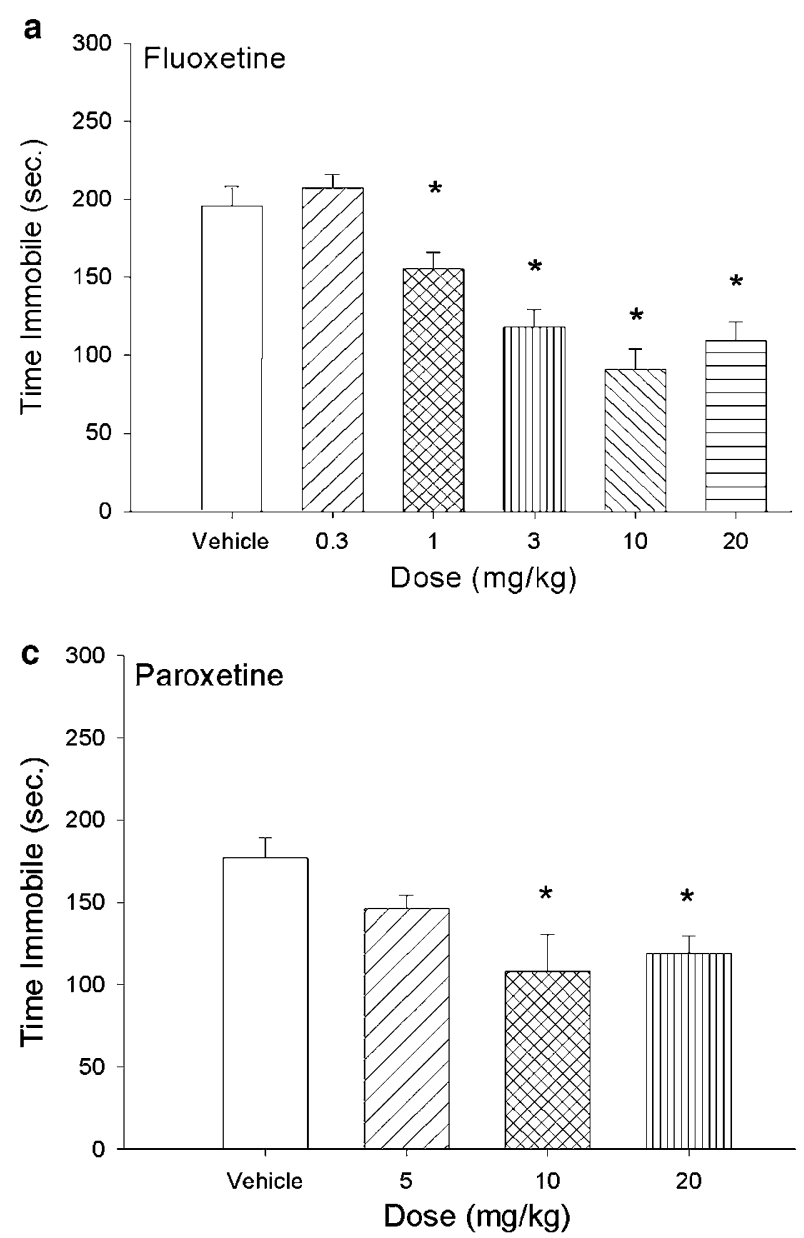

In addition to tank diameter, it was observed that gerbils weighing more than $55 \mathrm{~g}$ at the time of the swim test performed more reliably than smaller animals. Gerbils that weighed less than $55 \mathrm{~g}$ were not consistently able to complete the 6-min test without being rescued from drowning.

Sex differences were also apparent in the development of the gerbil FST. Male animals spent more time immobile (average immobility time $180 \mathrm{~s}$ ) as compared with female animals (average immobility $119 \mathrm{~s}$ ), and thus appeared more sensitive to the antidepressant-induced suppression of immobility when administered desipramine, paroxetine, or fluoxetine (data not shown). Therefore, male animals were used for all subsequent pharmacological validation. The alterations in immobility observed in male gerbils were inversely proportional to changes in swimming behavior almost exclusively. Unlike rats, gerbils did not show significant climbing behavior at any time in the test.

\section{Pharmacological Validation}

The SSRI compounds fluoxetine $(0.3-20 \mathrm{mg} / \mathrm{kg})$ and paroxetine $(5-20 \mathrm{mg} / \mathrm{kg})$ produced significant dose dependent decreases in immobility in the FST (Figure 1a and c). The minimum effective dose for fluoxetine $(\mathrm{F}(5,74)=16.691$;
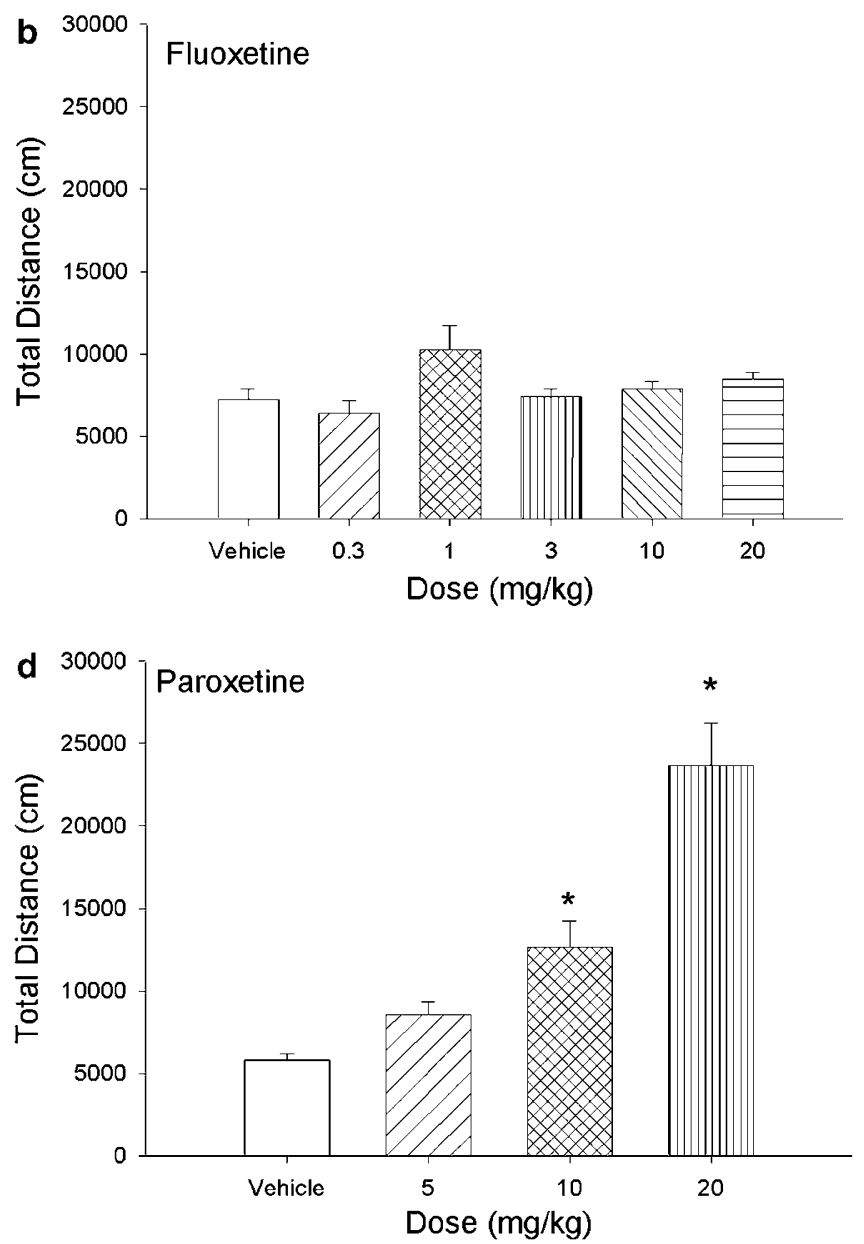

Figure I Behavioral effects of selective serotonin reuptake inhibitors in the gerbil forced swim and locomotor activity tests. Gerbils were administered either fluoxetine (0.3-20 mg/kg, p.o.) or paroxetine (5-20 mg/kg, p.o.) 60 min prior to testing. Fluoxetine: (a) forced swim; (b) locomotor activity; Paroxetine: (c) forced swim; (d) locomotor activity. $* p<0.05$ vs vehicle. $N=10$ per group. 
$p<0.0001)$ and paroxetine $(\mathrm{F}(3,36)=4.518 ; p<0.01)$ was 1.0 and $10.0 \mathrm{mg} / \mathrm{kg}$, respectively. Interestingly, whereas fluoxetine did not significantly alter LMA at any of the doses tested $(\mathrm{F}(5,55)=3.801 ; p<0.05)$ (Figure $1 \mathrm{~b})$, paroxetine produced a significant increase in total distance traveled at doses of 10 and $20 \mathrm{mg} / \mathrm{kg}(\mathrm{F}(4,43)=12.379$; $p<0.05$ ) (Figure 1d).

The tricyclic antidepressant desipramine $(3-30 \mathrm{mg} / \mathrm{kg})$ produced a dose-dependent decrease in immobility. The minimum effective dose was less than or equal to $3.0 \mathrm{mg} / \mathrm{kg}$
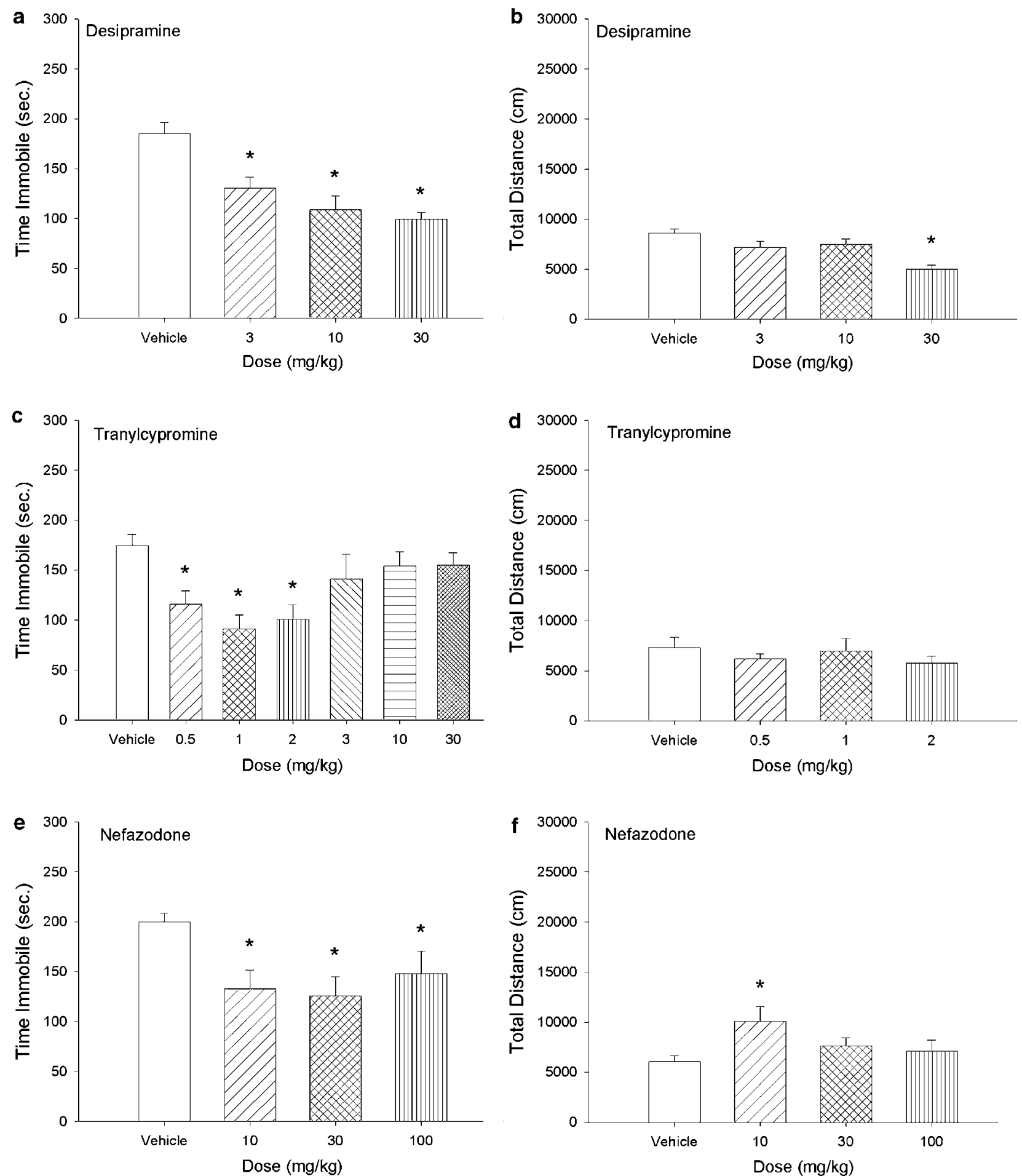

Figure 2 Behavioral effects of tricyclic antidepressants, monoamine oxidase inhibitors, and selective norepinephrine reuptake inhibitors in the gerbil forced swim and locomotor activity tests. Gerbils were administered desipramine (3-30 mg/kg, p.o.) tranylcypromine $(0.5-30 \mathrm{mg} / \mathrm{kg}, \quad$ p.o.), or nefazodone (10-100 mg/kg, p.o.) 60 min prior to testing. Desipramine: (a) forced swim; (b) locomotor activity; Tranylcypromine: (c) forced swim; (d) locomotor activity; Nefazodone: (e) forced swim; (f) locomotor activity. ${ }^{*} p<0.05$ vs vehicle. $N=10$ per group. 
$(\mathrm{F}(3,36)=12.128 ; \quad p<0.001) \quad($ Figure 2a). Desipramine produced a significant reduction in LMA only at the highest dose tested $(30 \mathrm{mg} / \mathrm{kg})(\mathrm{F}(4,42)=0.0051 ; p<0.05)$, whereas lower doses $(3.0$ and $10 \mathrm{mg} / \mathrm{kg})$ selectively decreased immobility in the FST (Figure 2b).

Low doses of the monoamine oxidase inhibitor tranylcypromine $(0.5-2 \mathrm{mg} / \mathrm{kg})$ decreased immobility time in the swim test, whereas higher doses did not alter immobility times as compared to vehicle-treated gerbils $(\mathrm{F}(6,73)=4.710 ; p<0.001)$ (Figure $2 \mathrm{c})$. In the LMA test, none of the doses of tranylcypromine tested significantly altered total distance traveled as compared to vehicle $(\mathrm{F}(4,41)=0.0001 ; p<0.05)$ (Figure $2 \mathrm{~d}$ )

Nefazodone, a selective norepinephrine reuptake inhibitor, administered at doses of 10,30 , and $100 \mathrm{mg} / \mathrm{kg}$ reduced immobility in the FST as compared to vehicle-treated animals $(\mathrm{F}(3,36)=0.0250 ; p<0.05)$ (Figure $2 \mathrm{e})$. Interestingly, the lowest dose of nefazodone tested (ie $10 \mathrm{mg} / \mathrm{kg}$ ) significantly increased total distance traveled in the locomotor assay, whereas the higher doses tested (ie 30 and $100 \mathrm{mg} / \mathrm{kg}$ ) produced no significant effect $v s$ the vehicle group $(\mathrm{F}(4,40)=0.0295 ; p<0.05)$ (Figure $2 \mathrm{f}$ ).

The psychostimulant, amphetamine produced a dosedependent decrease in immobility in the FST, with all doses tested $(1,3$, and $10 \mathrm{mg} / \mathrm{kg}$ ) producing a significant effect $(\mathrm{F}(3,36)=0.001 ; p<0.05)$ (Table 2). The decrease in immobility produced by amphetamine was paralleled by an increase in LMA, with the $10 \mathrm{mg} / \mathrm{kg}$ dose producing a significant effect $(\mathrm{F}(3,34)=0.036 ; p<0.05)$.

The antipsychotic drug haloperidol increased immobility time in the gerbil FST at 1.0 and $3.0 \mathrm{mg} / \mathrm{kg}$, whereas the lowest dose tested $(0.3 \mathrm{mg} / \mathrm{kg})$ did not significantly alter immobility $(\mathrm{F}(3,36)=0.0001 ; p<0.05)$ (Table 2$)$. The increase in immobility produced by haloperidol was paralleled by a decrease in LMA with the two highest doses tested $(1.0$ and $3.0 \mathrm{mg} / \mathrm{kg})$ producing significant decreases $(\mathrm{F}(4,39)=0.0001 ; p<0.05)$.

Table 2 Behavioral Effects of Additional Psychotropic Agents

\begin{tabular}{lccc} 
Treatment & Dose & Immobility (s) & Total distance (cm) \\
\hline Amphetamine & 0 & 194.6 & $5372 \pm 652$ \\
& 1 & $135.4^{*}$ & $7215 \pm 792$ \\
Haloperidol & 3 & $114.6^{*}$ & $7913 \pm 742$ \\
& 10 & $35.8^{*}$ & $12429 \pm 2265^{*}$ \\
Diazepam & 0 & 162.6 & $7572 \pm 599$ \\
& 0.3 & 175.8 & $6681 \pm 666$ \\
& 1 & $244.4 *$ & $4316 \pm 658^{*}$ \\
Buspirone & 3 & $250.2^{*}$ & $1112 \pm 135^{*}$ \\
& 0 & 167.0 & $6735 \pm 474$ \\
& 0.1 & 209.7 & $8758 \pm 1067$ \\
& 1 & 211.2 & $8524 \pm 986$ \\
& 0 & $249.1 *$ & $1 \mid 213 \pm 885^{*}$ \\
& 3 & 180.6 & $8086 \pm 802$ \\
& 10 & 178.2 & $7218 \pm 97 \mid$ \\
30 & 177.5 & $7720 \pm 977$ \\
& 173.1 & $470 \pm 324^{*}$ \\
\hline
\end{tabular}

* $p<0.05$ as compared to vehicle treated group.
The benzodiazepine, diazepam, significantly increased immobility in the forced swim and in the LMA tests at $1.0 \mathrm{mg} / \mathrm{kg}$, but had no effect at 0.1 and $0.3 \mathrm{mg} / \mathrm{kg}$ doses in either test (FST: $\mathrm{F}(3,36)=0.0115 ; p<0.05)$ (LMA: $\mathrm{F}(4,37)=0.0001 ; p<0.05)$ (Table 2).

A second anxiolytic compound, buspirone (a $5-\mathrm{HT}_{1 \mathrm{~A}}$ agonist), was also tested in the FST. Buspirone $(3,10$, or $30 \mathrm{mg} / \mathrm{kg}$ ) produced no significant alterations in immobility in this test $(\mathrm{F}(3,36)=0.9130)$ (Table 2). In addition, buspirone had no effect on total distance traveled when administered at 3 or $10 \mathrm{mg} / \mathrm{kg}$, but did produce a significant decrease in LMA at the highest dose tested (ie $30 \mathrm{mg} / \mathrm{kg}$ ) $(\mathrm{F}(4,41)=0.0001 ; p<0.05)$.

\section{Effects of NK1 Antagonists}

To determine whether NK1 receptor antagonists would exhibit antidepressant-like effects in the gerbil FST, three prototypic compounds (eg MK-869, L-733060, CP-122721) that have previously been reported to exhibit behavioral effects (Duffy et al, 2002; Varty et al, 2002b, 2003) were tested in the gerbil forced swim and LMA tests. MK-869 $(0.1-30 \mathrm{mg} / \mathrm{kg})$ dose dependently reduced immobility times, with a minimum effective dose of $1 \mathrm{mg} / \mathrm{kg}$ $(\mathrm{F}(6,93)=26.257 ; p<0.05)$ (Figure 3a). MK-869 did not alter LMA at any of the doses tested $(F(7,87)=2.238$; $p<0.05$ ) (Figure 3b). The NK1 receptor antagonist L-733060 similarly reduced immobility in the FST, but only at the highest doses tested (10 and $30 \mathrm{mg} / \mathrm{kg})(\mathrm{F}(3,36)=18.031$; $p<0.05$ ) (Figure $3 \mathrm{c}$ ) without producing any changes in total distance traveled $(\mathrm{F}(4,41)=3.201 ; p<0.05)$ (Figure $3 \mathrm{~d}$ ). Finally, CP-122721 (0.3-30 mg/kg) produced a dose dependent decrease in immobility in the gerbil FST with a minimum effective dose of $3 \mathrm{mg} / \mathrm{kg}(\mathrm{F}(5,63)=19.402$; $p<0.05)$ (Figure $3 \mathrm{e}$ ). CP-122721 did not alter total distance traveled at any of the doses tested $(\mathrm{F}(4,42)=2.591 ; p<0.05)$ (Figure 3f).

\section{DISCUSSION}

In the present study, we have adapted the FST, a widely employed model for identifying antidepressant-like activity in rodents, for use in the gerbil. Whereas the fundamental principles of the FST as previously demonstrated in rats and mice remained consistent, several technical factors were modified to ensure reliability and predictability when used in the gerbil.

It was identified relatively early in the model development that the size of the swim tank was a critical factor. More specifically, increasing the diameter of the swim tank enabled the gerbils to complete the swim test. It has been reported previously that the effects of the swim tank area can affect reliability of forced-swim data (Sunal et al, 1994). Consistent with this report, the present study indicated that the performance of the gerbils in the forced-swim environment was more reliable and more sensitive to antidepressant drug treatment when the diameter of the swim tank was larger (ie $50 \mathrm{~cm}$ ) than when tested in smaller containers (eg 15 or $30 \mathrm{~cm}$ ). Moreover, it was noted that in the smaller diameter swim tanks tested, gerbils, unlike mice and rats, 

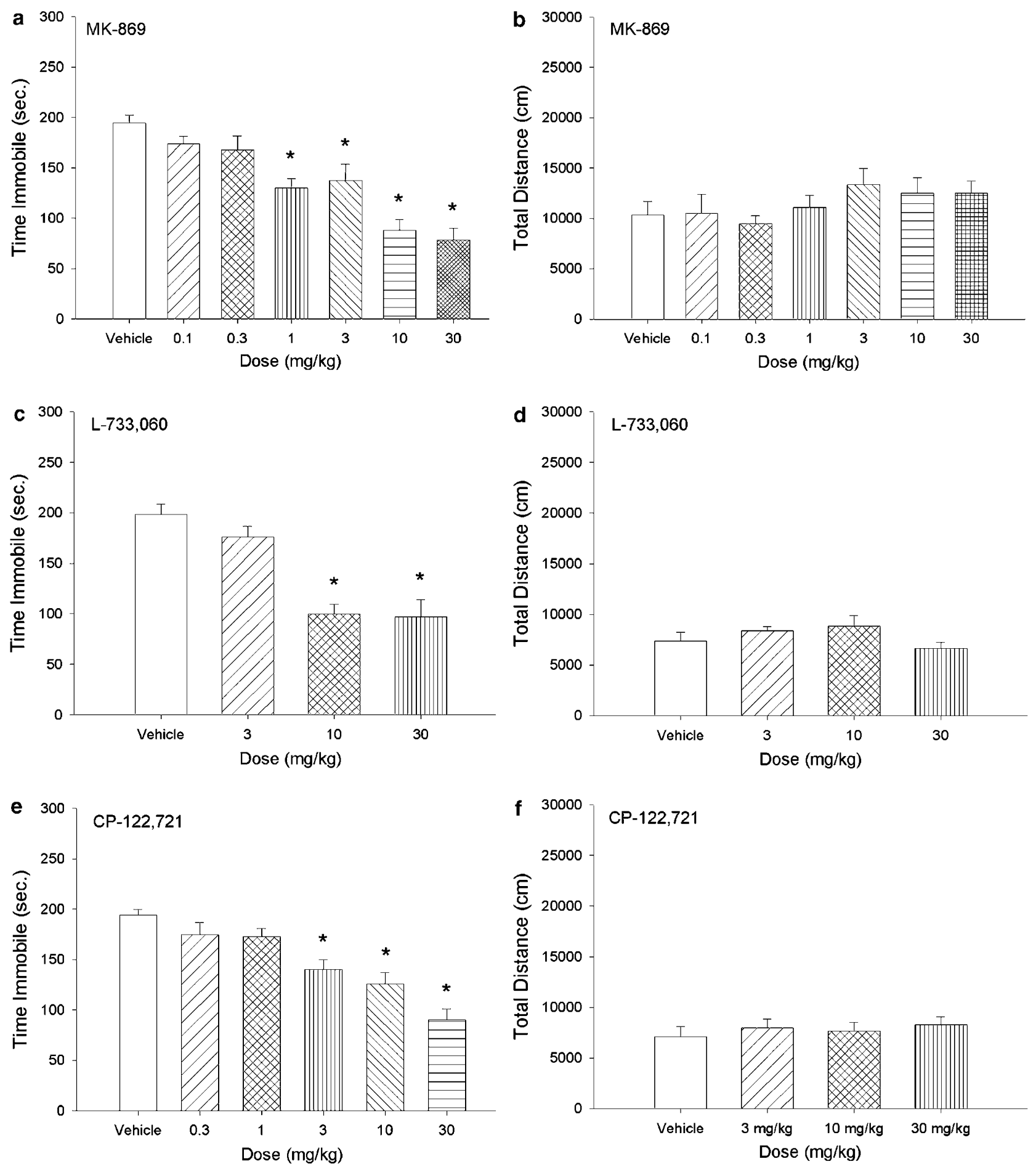

Figure 3 Behavioral effects of neurokinin-I receptor antagonists in the gerbil-forced swim and locomotor activity tests. Gerbils were administered MK-869 (0. I-30 mg/kg, p.o.), L-733060 (3-30 mg/kg, p.o.), or CP-I2272I (3-30 mg/kg, p.o.) I 20 min prior to testing. MK-869: (a) forced swim; (b) locomotor activity; L-733060: (c) forced swim; (d) locomotor activity; CP-I2272I: (e) forced swim; (f) locomotor activity. $* p<0.05$ vs vehicle. $N=10$ per group.

have difficulty completing the swim session altogether and require continuous monitoring to prevent drowning.

In addition to the swim tank diameter, the weight of the gerbils was also determined to be a critical factor in assessing the reliability of the assay. Heavier weight gerbils (ie greater than or equal to $55 \mathrm{~g}$ ) performed better than lighter weight gerbils (ie less than $55 \mathrm{~g}$ ). This phenomenon appears to be independent of sex in that both male and female gerbils weighing at least $55 \mathrm{~g}$ were able to successfully complete the $6 \mathrm{~min}$ swim session. The $55 \mathrm{~g}$ weight is reached at approximately 10-12 weeks of age for males and $12+$ weeks for females, which corresponds to adulthood in the Mongolian gerbil. Whereas more investigation into the weight differences in the gerbils is warranted, based on 
observation, the lighter weight gerbils appeared to fatigue much sooner than heavier animals and thus were not successful at completing the test session without having to be rescued. It is possible that the muscle mass that is present at an earlier age/lighter weight is not sufficient to enable the animals to complete the strenuous swim test. As the gerbils age and body mass increases, these changes are hypothesized to enable them to maintain active behaviors throughout the 6-min session. Alternatively and/or in conjunction with increased muscle mass, an increase in body fat as the gerbils age may provide the buoyancy and/or additional energy needed to stay afloat during the swim session.

It has been reported previously that rats exhibit distinct active behaviors, namely swimming and climbing, after administration of compounds that selectively inhibit the reuptake of serotonin or norepinephrine systems, respectively (Detke et al, 1995). The active behaviors have been replicated in several laboratories, and are inversely proportional to the total immobility score, such that a drug that increases swimming or climbing will concomitantly produce a decrease in immobility. In the present study, this distinction in active behaviors (swimming $v s$ climbing) in gerbils was not readily identifiable in the large swim tank (ie $50 \mathrm{~cm}$ ). Gerbils tended to swim or were immobile with very little climbing behavior observed. The climbing behavior may have been more apparent in a smaller diameter tank, ie a tank more similar in size to a rat swim tank $($ eg $20 \mathrm{~cm})$; however, as noted previously, gerbils had difficulty completing the 6-min swim session in the smaller tanks and behavior could not be reliably scored.

In the present studies, male gerbils showed a more consistent reduction in immobility with antidepressant compounds in the FST than female animals. In general, it was observed that female vehicle-treated gerbils tended to exhibit much less inactivity (average immobility time 119s) than male gerbils (average immobility time $180 \mathrm{~s}$ ) during the $6 \mathrm{~min}$ session, making the expected increase in active behaviors with antidepressants difficult to distinguish from control-treated animals. Whereas most of the preclinical work investigating the effects of antidepressant compounds in rodent models of depression use male animals, several reports in which female animals were studied suggest that significant sex differences are apparent. More specifically, Brotto et al (2000) have reported an increase in activity levels of female rats as compared to male rats in the FST similar to the data shown in the present gerbil study. The sex dependent effects may be in part due to the phase of the estrous cycle in which female animals are tested (Barros and Ferigolo, 1998; Alonso et al, 1991; Kennett et al, 1986). In the FST, in particular, female rats exhibit less immobility than their male counterparts during the diestrous phase of the hormonal cycle, whereas during the proestrous phase, female rats show levels of immobility similar to male rats (Barros and Ferigolo, 1998). As changes in the estrous cycle were not evaluated in the present study, the impact that estrogen and progesterone may have on female gerbil behavior in the FST remains to be evaluated.

To validate the gerbil FST, several antidepressants and compounds from other therapeutic classes were used. Compounds previously reported to produce antidepressant-like activity both preclinically and clinically were active in this paradigm. Both SSRIs tested (ie fluoxetine and paroxetine), as well as the tricyclic, desipramine, the monoamine oxidase inhibitor, tranylcypromine, and the SNRI, nefazodone, dose-dependently decreased immobility in the FST indicative of an antidepressant-like effect. It is interesting to note that several of the antidepressant compounds tested were effective at reducing immobility in the gerbil FST at much lower doses as compared to those typically reported for mice and rats (Porsolt et al, 1977a, b; 1978a,b; Page et al, 1999; Karolewicz and Paul, 2001; Bianchi et al, 2002; Crowley et al, 2004), although it is difficult to make direct comparisons because of the different routes of administration used in the present study (ie oral) $v s$ many of the other studies (eg i.p.). Overall, these data are suggestive that the gerbil may be more sensitive species to psychoactive substances compared to mice and rats.

Changes in immobility in the FST can be confounded by drug-induced alterations in LMA (Porsolt et al, 1977a). Therefore, all compounds evaluated in the forced-swim model were also evaluated for their potential effects on general motor activity. Interestingly, whereas fluoxetine had no effects on LMA at any of the doses tested, paroxetine significantly increased the total distance traveled in the LMA test. The paroxetine-induced increase in LMA occurred at doses that reduced immobility in the FST, and therefore, could not be dissociated from its apparent antidepressant-like efficacy. Sertraline and citalopram have similarly been reported to increase hyperlocomotion in gerbils, although these studies did not report alterations in LMA with paroxetine (Varty et al, 2002a, 2003). The increased activity induced by some SSRI compounds should not be generalized across this class of molecules, since fluoxetine has consistently been shown to not increase LMA in gerbils in (present study; Varty et al (2002a)).

Several other classes of antidepressant compounds tested in the present study were also assessed for the potential of changes in LMA to confound the interpretation of FST behavior. The tricyclic antidepressant, desipramine, significantly reduced immobility at doses that were similar to those reported to be active in rats and mice. At the highest dose tested (ie $30 \mathrm{mg} / \mathrm{kg}$ ), desipramine produced a significant decrease in LMA, consistent with results reported for this compound in rats (Porsolt et al, 1978a). However, a decrease in activity would not be expected to influence the reduction in immobility. Moreover, a dissociation between effects on LMA and immobility was observed with desipramine at the two lower doses tested (3 and $10 \mathrm{mg} /$ $\mathrm{kg}$ ), both of which reduced immobility without affecting LMA. Similarly, tranylcypromine did not exhibit any alterations in LMA at any of the efficacious doses tested, although higher doses $(3,10$ and $30 \mathrm{mg} / \mathrm{kg})$ did reduce LMA such that gerbils could not complete the swim test without being rescued. The SNRI nefazodone showed significant reductions in immobility in the FST at all doses tested. The lowest dose of nefazodone also showed significant locomotor-stimulating properties in the gerbil. However, the increase in total distance traveled was not observed with higher doses of nefazodone, whereas the antidepressant-like effects were suggesting that the decrease in immobility produced by nefazodone was not entirely due to an increase in LMA. The antidepressant-like effects of nefazodone observed in the present study have been demonstrated 
previously using the tail suspension test (Varty et al, 2003); however, to our knowledge, this is the first report of this compound's activity in the FST.

Consistent with previous reports that anxiolytic agents do not produce antidepressant effects in the FST (Porsolt et al, 1977a, b, 1978a; Lucki, 1997; Varty et al, 2003), buspirone had no effect on time spent immobile in the FST. Interestingly, buspirone was previously reported to exhibit antidepressant-like properties in rats in the FST (Wieland and Lucki, 1990); however, this effect was seen only after subchronic administration. The present study used acute administration, and this difference may be one reason why antidepressant-like effects were not observed with buspirone in gerbils. Whereas the highest doses of buspirone did not alter FST behavior, the highest dose of diazepam tested significantly increased immobility in the FST. Moreover, buspirone and diazepam at the highest doses tested significantly decreased or increased total distance traveled, respectively. The latter finding suggests that increased LMA alone is not sufficient to result in antidepressant-like effects in this model. In gerbils, higher doses of diazepam ( 3 and $10 \mathrm{mg} / \mathrm{kg}$ ) produced significant decreases in LMA and were therefore not tested in the FST because of predicted debilitating effects in this assay (personal observation).

The psychostimulant, amphetamine, reduced immobility in the gerbil FST at all doses tested; however, this effect may be due to the motor-stimulating properties $v s$ the escapeoriented behaviors of this drug. Amphetamine has consistently been reported to decrease immobility in both the mouse and rat FSTs (Porsolt et al, 1977b, 1978a) at similar doses to those used in the present study. However, the locomotor-stimulating effects of this psychostimulant have made it difficult to determine whether or not amphetamine has antidepressant-like effects in this assay (Borsini and Meli, 1988).

In contrast to amphetamine, the neuroleptic compound, haloperidol, significantly increased immobility in the gerbil FST and decreased total distance traveled in the LMA test at the highest doses tested ( 1 and $3 \mathrm{mg} / \mathrm{kg}$ ). The lowest dose of haloperidol tested $(0.3 \mathrm{mg} / \mathrm{kg})$ did not produce any noticeable effects in either the FST or motor test. These results are consistent with the recognized cataleptic properties of haloperidol in rats and mice (Costall and Naylor, 1975; Zetler and Baumann, 1985), and demonstrate the sensitivity of both assays to alterations in motor function.

The studies discussed above have shown that, like rat and mouse FST, gerbil FST is sensitive to classical antidepressants. NK1 antagonists have recently been proposed as a novel class of antidepressants that may have reduced side effects compared with classical treatments (Kramer et al, 1998). As gerbils show greater similarity with human NK1 receptor pharmacology compared with rats or mice, the gerbil is an ideal candidate for testing the behavioral effects of NK1 receptor antagonists (Beresford et al, 1991; Gitter et al, 1991). The present studies also investigated behavioral effects of NK1 antagonists. All three NK1 antagonists tested, MK-869, L-733060, and CP-122721, dose dependently decreased immobility in the gerbil FST without altering LMA, indicative of an antidepressant-like effect. The maximal behavioral response exhibited by MK-869, L733060 , and CP-122721 was approximately a 50\% reduction in immobility as compared to vehicle-treated animals, and was similar to behavioral responses observed after standard antidepressant (eg fluoxetine, desipramine) treatment in this model. Of the three NK1 antagonists tested, MK-869 exhibited the greatest potency by reducing immobility at $1 \mathrm{mg} / \mathrm{kg}$ after oral administration, followed by CP-122721 with a least effective dose of $3 \mathrm{mg} / \mathrm{kg}$, and L-733060 showing behavioral activity at $10 \mathrm{mg} / \mathrm{kg}$. Whereas these data are consistent with previously reported studies in which NK1 antagonists have been tested in the tail suspension test (Varty et al, 2003), the least effective dose for MK-869, L-733060 and CP-122721 was at least 10 times lower in the gerbil FST with brain exposures of these compounds identified as being dose dependent (not shown) as compared to the gerbil tail suspension model. These data suggest that the FST may be a more sensitive paradigm for identifying the behavioral effects of NK1 receptor antagonists.

In the present study, the preclinical antidepressant-like effect observed with NK1 receptor antagonists is comparable to that of numerous clinically approved antidepressant drugs, and suggests a role for NK1 receptor antagonists in the treatment of depression. However, in a recent Phase III clinical trial, MK-869 failed to demonstrate adequate clinical antidepressant activity (Keller et al, 2006). Whether this lack of antidepressant efficacy can be generalized to other NK1 antagonists remains to be established. The results of Keller et al (2006) are in marked contrast to the results of the present study, as well as previously reported preclinical data (Kramer et al, 1998; Varty et al, 2003), and promising antidepressant results in a phase II clinical trial of MK-869 (Kramer et al, 1998). It is unclear at this time why such discrepancies exist; however, more extensive clinical testing will need to be conducted to better understand the potential use of NK1 antagonists in the treatment of depression as well as to determine the relevance of the gerbil FST in predicting clinical efficacy. As with most preclinical antidepressant tests, the gerbil FST does not model depression itself; thus, caution should be exercised when interpreting the data and additional de-risking exercises (eg assessment in disease relevant models) should be considered in conjunction with the FST when appropriate to minimize the potential for false positive results.

In summary, the present study reports successful adaptation of the FST for use in the gerbil. Whereas the fundamental principles of the FST as previously demonstrated in rats and mice remained the same, alterations in the size of the swim tank, the use of male animals, as well as animals weighing more than $55 \mathrm{~g}$ ensured greater reliability and predictability of the gerbil FST. The data presented in this study demonstrate pharmacological specificity for identifying standard antidepressant compounds, and suggest that the gerbil model performs comparably to models using more traditionally employed laboratories animals (ie rats, mice). In addition, the gerbil may be a more sensitive species for the identification of antidepressant compounds, in that the effects of standard antidepressants were detected at much lower doses than typically observed with rats or mice. Finally, NK1 receptor antagonists potently reduced immobility times in the gerbil FST at lower doses than have been previously reported in other tests sensitive to antidepressant activity (eg tail suspension). Overall, these data suggest that the gerbil FST may be a valuable tool for identifying novel antidepressant compounds. 


\section{ACKNOWLEDGEMENTS}

The authors thank Dr Snjezana Lelas for insightful comments provided during the preparation of this manuscript, and Bristol-Myers Squibb Discovery Chemistry for the synthesis of the NK1 antagonists.

\section{DISCLOSURE}

All financial and material support for the work presented in this manuscript was provided by Bristol-Myers Squibb. There are no other relevant financial interests to disclose.

\section{REFERENCES}

Alonso SJ, Castellano MA, Afonso D, Rodriguez M (1991). Sex differences in behavioral despair: relationships between behavioral despair and open field activity. Physiol Behav 49: 69-72.

Ballard TM, Sangar S, Higgins GA (2001). Inhibition of shockinduced foot tapping behaviour in there gerbil by a tachykinin NK1 receptor antagonist. Eur J Pharmacol 412: 255-264.

Barros HMT, Ferigolo M (1998). Ethopharmacology of imipramine in the forced-swimming test: gender differences. Neurosci Biobehav Rev 23: 279-286.

Beresford IJ, Birch PJ, Hagan RM, Ireland SJ (1991). Investigation into species variants in tachykinin NK1 receptors by use of the non-peptide antagonist, CP-96, 345. Br J Pharmacol 104: 292-293.

Bianchi M, Moser C, Lazzarani C, Vecchiato E (2002). Forced swimming test and fluoxetine treatment: in vivo evidence that peripheral 5-HT in rat platelet-rich plasma mirrors cerebral extracellular 5-HT levels, whilst 5-HT in isolated platelets mirrors neuronal 5-HT changes. Exp Brain Res 143: 191-197.

Borsini F, Meli A (1988). Is the forced swimming test a suitable model for revealing antidepressant activity? Psychopharmacology 94: $147-160$

Brotto LA, Barr AM, Gorzalka BB (2000). Sex differences in forcedswum and open-field test behaviours after chronic administration of melatonin. Eur J Pharmacol 402: 87-93.

Costall B, Naylor RJ (1975). Detection of the neuroleptic properties of clozapine, sulpiride and thioridazine. Psychopharmacologia 43: 69-74.

Crowley JJ, Jones MD, O'Leary OF, Lucki I (2004). Automated tests for measuring the effects of antidepressants in mice. Pharmacol Biochem Behav 78: 269-274.

Cryan JF, Markou A, Lucki I (2002). Assessing antidepressant activity in rodents: recent developments and future needs. Trends Pharmacol Sci 23: 238-245.

Detke MJ, Johnson J, Lucki I (1997). Acute and chronic antidepressant drug treatment in the rat forced swimming test model of depression. Exp Clin Psychopharmacol 5: 107-112.

Detke MJ, Rickels M, Lucki I (1995). Active behaviors in the rat forced swimming test differentially produced by serotonergic and noradrenergic antidepressants. Psychopharmacology 121: 66-72.

Duffy RA, Varty GB, Morgan CA, Lachowicz JE (2002). Correlation of neurokinin (NK) 1 receptor occupancy in gerbil striatum with behavioral effects of NK1 antagonists. J Pharmacol Exp Ther 301: 536-542.

File SE, Cheeta S, Akanezi C (2001). Diazepam and nicotine increase social interaction in gerbils: a test for anxiolytic action. Brain Res 888: 311-313.

Gentsch C, Cutler M, Vassout A, Veenstra S, Brugger F (2002). Anxiolytic effect of NKP608, a NK1-receptor antagonist, in the social investigation test in gerbils. Behav Brain Res 133: 363-368.
Gitter BD, Waters DC, Bruns RF, Mason NR, Nixon JA, Howbert JJ (1991). Species differences in affinities of non-peptide antagonists for substance P receptors. Eur J Pharmacol 197: 237-238.

Karolewicz B, Paul IA (2001). Group housing of mice increases immobility and antidepressant sensitivity in the forced swim and tail suspension tests. Eur J Pharmacol 415: 197-201.

Keller M, Montgomery S, Ball W, Morrison M, Snavely D, Liu G et al (2006). Lack of efficacy of the substance P (neurokinin 1 receptor) antagonist aprepitant in the treatment of major depressive disorder. Biol Psychiatry 59: 216-223.

Kennett GA, Chaouloff F, Marcou M, Curzon G (1986). Female rats are more vulnerable than males in an animal model of depression: the possible role of serotonin. Brain Res 382: 416-421.

Kramer MS, Cutler N, Feighner J, Shrivastava R, Carman J, Sramek JJ et al (1998). Distinct mechanism for antidepressant activity by blockade of central substance $\mathrm{P}$ receptors. Science 281: $1640-1645$.

Lucki I (1997). The forced swimming test as a model for core and component behavioral effects of antidepressant drugs. Behav Pharmacol 8: 523-532.

Lucki I, Dalvi A, Mayorga AJ (2001). Sensitivity to the effects of pharmacologically selective antidepressants in different strains of mice. Psychopharmacology 155: 315-322.

Maier SF, Seligman MEP (1976). Learned helplessness: theory and evidence. J Exp Psychol 105: 3-46.

Nestler EJ, Gould E, Manji H, Duman RS, Greshenfeld HK, Hen R et al (2002). Preclinical models: status of basic research in depression. Biol Psychiatry 52: 503-528.

Page ME, Detke MJ, Dalvi A, Kirby LG, Lucki I (1999). Serotonergic mediation of the effects of fluoxetine, but not desipramine, in the rat forced swimming test. Psychopharmacology 147: 162-167.

Porsolt RD, Anton G, Blavet N, Jalfre M (1978a). Behavioural despair in rats: a new model sensitive to antidepressant treatments. Eur J Pharmacol 47: 379-391.

Porsolt RD, Bertin A, Jalfre M (1977b). Behavioral despair in mice: a primary screening test for antidepressants. Arch Int Pharmacodyn Ther 229: 327-336.

Porsolt RD, Bertin A, Jalfre M (1978b). 'Behavioural despair' in rats and mice: strain differences and the effects of imipramine. Eur J Pharmacol 51: 291-294.

Porsolt RD, Pichon ML, Jalfre M (1977a). Depression: a new animal model sensitive to antidepressant treatments. Nature 266: 730-732.

Rupniak NMJ, Carlson EJ, Webb JK, Harrison T, Porsolt RD, Roux $S$ et al (2001). Comparison of the phenotype of NK1R-I- mice with pharmacological blockade of the substance $\mathrm{P}$ (NK1) receptor in assays for antidepressant and anxiolytic drugs. Behav Pharmacol 12: 497-508.

Rupniak NMJ, Webb JK, Fisher A, Smith D, Boyce S (2003). The substance P (NK1) receptor antagonist L-760735 inhibits fear conditioning in gerbils. Neuropharmacology 44: 516-523.

Santarelli L, Gobbi G, Debs PC, Sibille EL, Blier P, Hen R et al (2001). Genetic and pharmacological disruption of neurokinin 1 receptor function decreases anxiety-related behaviors and increases serotonergic function. Proc Natl Acad Sci USA 98: 1912-1917.

Steru L, Chermat R, Thierry B, Simon P (1985). The tail suspension test: a new method for screening antidepressants in mice. Psychopharmacology 85: 367-370.

Sunal R, Grumusel B, Kayaalp SO (1994). Effect of changes in swimming area on results of 'behavioral despair test'. Pharmacol Biochem Behav 49: 891-896.

Varty GB, Cohen-Williams ME, Hunter JC (2003). The antidepressant-like effects of neurokinin NK1 receptor antagonists in a gerbil tail suspension test. Behav Pharmacol 14: 87-95. 


\section{Gerbil forced-swim test}

TL Wallace-Boone et al

Varty GB, Cohen-Williams ME, Morgan CA, Pylak U, Duffy RA, Lachowicz JE et al (2002b). The gerbil elevated plus-maze II: anxiolytic-like effects of selective neurokinin NK1 receptor antagonists. Neuropsychopharmacology 27: 371-379.

Varty GB, Morgan CA, Cohen-Williams ME, Coffin VL, Carey GJ (2002a). The gerbil elevated plus-maze I: behavioral characterization and pharmacological validation. Neuropsychopharmacology 27: 357-370.
Wieland S, Lucki I (1990). Antidepressant-like activity of 5-HT1A agonists measured with the forced swim test. Psychopharmacology 101: 497-504.

Wong ML, Licinio J (2004). From monoamines to genomic targets: a paradigm shift for drug discovery in depression. Nat Rev Drug Discov 3: 136-151.

Zetler G, Baumann GH (1985). Pharmacokinetics and effects of haloperidol in the isolated mouse. Pharmacology 31: 318-327. 